\title{
TUBERCULOSE RESISTENTE E MULTIRRESISTENTE NO BRASIL
}

\author{
Resistant and Multidrug-Resistant Tuberculosis in Brazil
}

\author{
Caroline Coelho de Oliveira ${ }^{1}$ \\ Eduarda Martins Cruz ${ }^{2}$ \\ Gabriela Drummond Magalhães ${ }^{3}$ \\ Mariana Veloso Suzart ${ }^{4}$ \\ Mateus Augusto de Prince ${ }^{5}$ \\ Camila Teles Gonçalves ${ }^{6}$ \\ Jaqueline Teixeira Teles Gonçalves ${ }^{7}$ \\ Luçandra Ramos Espírito-Santo ${ }^{8}$ \\ Carlos Eduardo Mendes D'Angelis ${ }^{9}$ \\ Karina Andrade de Prince ${ }^{10}$
}

\footnotetext{
${ }^{1}$ Graduada de Medicina pelo Centro Universitário FIPMoc - (UNIFIPMoc). Montes Claros/MG-Brasil. $\bowtie$ carolinecoelhodeoliveira@gmail.com 9 https://orcid.org/0000-0002-6151-676X.

${ }^{2}$ Graduada de Medicina pelo Centro Universitário FIPMoc - (UNIFIPMoc). Montes Claros/MG-Brasil. martinseduaeda88@gmail.com. (1) https://orcid.org/0000-0003-0492-7391.

${ }^{3}$ Universidade Estadual de Montes Claros (UNIMONTES), Programa de Pós-graduação em Cuidados Primários em Saúde. Montes Claros, MG - Brasil. $\square$ gabriela.gdm@hotmail.com. (1) https://orcid.org/0000-0001-95808261.

${ }^{4}$ Graduada de Medicina pelo Centro Universitário FIPMoc - (UNIFIPMoc). Montes Claros/MG-Brasil. marianavsuzart@outlook.com. (9) https://orcid.org/0000-0002-2068-3852.

${ }^{5}$ Graduando de Medicina pelo Centro Universitário FUNORTE - (UNIFUNORTE). Montes Claros/MG-Brasil. $\triangle$ mateus.prince@soufunorte.com.br. 1 https://orcid.org/0000-0003-4900-7345.

${ }^{6}$ Graduada de Medicina pelo Centro Universitário FUNORTE - (UNIFUNORTE). Montes Claros/MG-Brasil. $\triangle$ camilatelesg@ hotmail.com. (1) https://orcid.org/0000-0002-1924-7746.

${ }^{7}$ Mestre em Cuidado Primário em Saúde (UNIMONTES). Professora da Universidade Estadual de Montes Claros (Unimontes). Minas Gerais. Brasil. $₫$ jaquelinettg@gmail.com. @ https://orcid.org/0000-00020-85784857.

${ }^{8}$ Doutora em Ciências da Saúde (UNIMONTES). Professora da Universidade Estadual de Montes Claros (Unimontes). Minas Gerais. Brasil. $₫$ la_lu_joao@hotmail.com. (1) https://orcid.org/0000-0003-4792-6962.

${ }^{9}$ Doutor em Ciências Farmacêuticas (FCFRP-USP). Professor da Universidade Estadual de Montes Claros (Unimontes). Minas Gerais. Brasil. $₫$ carlos.dangelis@unimontes.br. 1 https://orcid.org/0000-0003-05886149.

${ }^{10}$ Doutora em Biociências e Biotecnologia Aplicadas a Farmácia (UNESP). Professora do Centro Universitário FIPMoc - (UNIFIPMoc). Minas Gerais. Brasil. $₫$ karina.prince@professor.unifipmoc.edu.br. https://orcid.org/0000-0001- 8231-852X.
}

$\begin{array}{ccc}\text { Recebido em } & \text { Aceito em } & \text { Publicado em } \\ 14 / 07 / 2021 & 04 / 08 / 2021 & 09 / 11 / 2021\end{array}$


Resumo: Objetivo: Analisar os casos de tuberculose resistente e multirresistente no Brasil, no período de 2009 a 2018. Métodos: Trata-se de um estudo, retrospectivo, descritivo, quantitativo, de base documental. Teve como universo de pesquisa a base de dados do Sistema de Informações de Agravos de Notificação (SINAN). Resultados: No Brasil, foram notificados 1795 casos de tuberculose resistente e 1577 de multirresistente. O número de casos aumentou expressivamente entre 2010 e 2017. Teve predomínio no sexo masculino ( $71,47 \%)$, na faixa etária entre $25-44$ anos $(41 \%)$, com $1^{\circ}$ grau incompleto $(43,44 \%)$, nas raças branca $(37,6 \%)$ e parda $(36,84 \%)(\mathrm{p}<0,001)$. Os casos novos $(45,97 \%)$ e de reingresso após abandono $(28,34 \%)$, apresentaram os maiores percentuais ( $p<0,001)$. Indivíduos alcoolistas ( $\mathrm{p} 0,002)$, portadores de HIV ( $<<0,001)$, com a forma pulmonar ( 0 0,023), e com abandono do tratamento ( $<<0,001)$, tiveram maiores percentuais de resistência adquirida. No entanto, portadores da forma extrapulmonar $(\mathrm{p} 0,023)$ e que evoluíram à cura, apresentaram maiores percentuais de resistência primária $(p<0,001)$. Conclusão: A tuberculose se mantém, como doença de alta incidência/prevalência no Brasil e, que apesar dos investimentos o percentual de casos de resistência permanece alto, trazendo importantes repercussões clínicas e epidemiológicas.

Palavras-Chave: Tuberculose; Mycobacterium tuberculosis; Tuberculose resistente a múltiplas drogas; Prevenção e controle.

\begin{abstract}
Objective: Analyze the cases of resistant and multidrug resistant tuberculosis in Brazil, from 2009 to 2018. Methods: This is a retrospective, descriptive, quantitative, document-based study. Its universe of research was the Data Base of the Information System for Notifiable Diseases (SINAN). Results: Were reported, in Brazil, 1795 cases of drug-resistant tuberculosis and 1577 of multidrug-resistant tuberculosis. The number of cases increased significantly between 2010 and 2017. There was a predominance in males $(71.47 \%)$, aged between $25-44$ years $(41 \%)$, with incomplete elementary school $(43.44 \%)$, in white races $(37.6 \%)$ and brown $(36.84 \%)(p<0.001)$. New cases (45.97\%) and re-entry after abandonment $(28.34 \%)$ had the highest percentages $(\mathrm{p}<0.001)$. Alcoholic individuals ( $\mathrm{p} 0.002)$, HIV carriers ( $\mathrm{p}<0.001)$, with the pulmonary form ( $\mathrm{p} 0.023)$, and with treatment dropout $(\mathrm{p}<0.001)$ had higher percentages of acquired resistance. However, patients with the extrapulmonary form ( $\mathrm{p}$ 0.023) and who evolved to cure had higher percentages of primary resistance ( $\mathrm{p}<0.001)$. Conclusion: Tuberculosis remains a high incidence/prevalence disease in our country and, despite the investments, the percentage of cases of resistance to first choice drugs remains high, bringing important clinical and epidemiological repercussions.
\end{abstract}

Keywords: Tuberculosis; Mycobacterium tuberculosis; Tuberculosis resistant to multiple drugs; Prevention and control.

\title{
INTRODUÇÃO
}

A tuberculose (TB) é uma doença infecciosa crônica causada por qualquer uma das sete espécies que integram o complexo Mycobacterium tuberculosis, sendo transmitida pela inalação de aerossóis da via aérea de um doente com TB ativa pulmonar ou laríngea. ${ }^{1}$ Possui alta letalidade, afetando principalmente populações de baixa renda, estando associada principalmente à falta de higiene e má alimentação. ${ }^{2}$ 
Estima-se que em 2015 cerca de 10,4 milhões de pessoas desenvolveram tuberculose (TB), 580 mil na forma de TB multirresistente (TB MDR) ou TB resistente à rifampicina (TB RR), e 1,4 milhão morreram da doença. ${ }^{1}$

$\mathrm{O}$ tratamento de $1^{\circ}$ linha para TB é dividido em uma primeira fase intensiva com dois meses de duração em que são utilizados rifampicina (RIF), isoniazida (INH), pirazinamida (PZA) e etambutol (EMB) e posteriormente a fase de manutenção por um período de quatro meses com RIF e INH. Esse esquema básico é muito eficaz na eliminação bacilar, desde que seja realizado adequadamente pelo paciente. ${ }^{3}$

O fenômeno da resistência bacilar foi identificado na década de 1940 quando foi descoberta a estreptomicina e instaurado seu uso no tratamento da tuberculose. São conhecidos três tipos de resistência: a natural, que surge naturalmente durante o processo de multiplicação da bactéria; a primária, quando o indivíduo é infectado por bacilos já resistentes aos medicamentos; e a resistência secundária ou adquirida que se caracteriza por pacientes com tuberculose que eram inicialmente sensíveis, mas que adquiriram resistência aos fármacos. $^{4,5}$

A tuberculose multirresistente (TB-MDR) é causada pelo Mycobacterium tuberculosis resistente a pelo menos rifampicina e à isoniazida. ${ }^{6}$ Sendo a rifampicina o medicamento mais ativo contra o bacilo de Koch, esquemas sem sua composição requerem o uso de fármacos de $2^{\circ}$ linha que resultam em tratamento com duração mais prolongada, com maior potencial de toxicidade, maiores chances de insucesso e de pior prognóstico. ${ }^{3}$

A resistência da bactéria aos medicamentos tem contribuído para aumentar a proporção de óbitos e recidivas da tuberculose. Os principais fatores associados a essa resistência são a monoterapia, prescrições impróprias e a falta de colaboração do paciente. A presença de multirresistência denuncia as deficiências no controle da TB e dificulta o tratamento e a prevenção. ${ }^{2}$

O expressivo número de pessoas infectadas pela tuberculose e por seu tratamento apresentar longa duração, acarreta o surgimento de cepas resistentes e multirresistentes. Assim, o objetivo do presente artigo foi analisar o número de casos de tuberculose resistente a drogas de $1^{\text {a }}$ linha e multirresistente no Brasil, no período de 2009 a 2018, bem como os fatores epidemiológicos associados. 


\section{MÉTODO}

Trata-se de um estudo, retrospectivo, descritivo, quantitativo, de base documental com procedimento comparativo-estatístico.

Teve como universo de pesquisa a base de dados do Sistema de Informação de Agravos de Notificação (SINAN), referente ao número de casos de tuberculose resistente de acordo com as regiões do Brasil, no período de 2009 a 2018. Os dados foram obtidos a partir do SINAN, disponibilizados pelo Departamento de Informática do SUS (DATASUS), no endereço eletrônico http://www2.datasus.gov.br).

Foram considerados como "resistentes" indivíduos que tiveram cepas isoladas de M. tuberculosis com resistência in vitro a pelo menos um dos fármacos de primeira linha (rifampicina, isoniazida, pirazinamida e etambutol) e como "multirresistente" indivíduos que apresentaram resistência a isoniazida e rifampicina. ${ }^{7}$ A resistência primária foi considerada entre os pacientes nunca tratados para tuberculose, infectados por cepas já resistentes aos medicamentos e, resistência adquirida doentes com tuberculose inicialmente droga sensível que se tornaram resistentes após a exposição aos fármacos. ${ }^{1}$

As variáveis estudadas foram: número de casos por ano e região, prevalência média por região, sociodemográficas (sexo, faixa etária, escolaridade e raça/cor), agravos associados (HIV, alcoolismo, uso de drogas ilícitas e tabagismo), tratamento diretamente observado (TDO) e dados epidemiológicos clínicos (ano notificação, tipo de entrada, forma da doença, situação de encerramento e tipo de resistência / primária ou adquirida).

Os dados populacionais para cálculo da prevalência média de casos de TB-MDR de acordo com as regiões do Brasil, foram obtidos a partir do portal do Instituto Brasileiro de Geografia e Estatística (IBGE).

Foi realizada análise descritiva das variáveis, com frequência, porcentagem e a média do número de casos registrados. A análise bivariada foi realizada por meio do teste Qui-quadrado de Pearson, para observar diferenças estatísticas entre as variáveis clínicas e os grupos que apresentavam resistência primária e resistência adquirida. Foi considerado como nível de significância, o valor de $\mathrm{p}<0,05$.

Utilizou-se o software Microsoft Office Excel® e o programa Statistical Pocckage for the Social Sciences (SPSS) para Windows, versão 25 (Chicago, IL, USA), para gerenciamento e análise de dados. 
Por se tratar de um banco de domínio público, não foi necessário submeter o projeto ao Comitê de Ética em Pesquisas.

\section{RESULTADOS}

De acordo com os dados epidemiológicos registrados no Sistema de Informação de Agravos de Notificação (SINAN), foram notificados 874.830 casos de tuberculose no Brasil, no período de 2009 a 2018. Desses 1795 (0,2\%), foram casos de resistência a drogas de $1^{\text {a }}$ linha e, $1577(0,18 \%)$ eram resistentes a isoniazida e rifampicina, caracterizando casos de tuberculose resistente a múltiplos medicamentos (TB-MDR) (Figura 1).

O número de casos de TB resistente a drogas de $1^{\text {a }}$ linha, variou de 25 a 359, com média de 179,5 casos. Observou-se um aumento expressivo no número de casos entre 2010 e 2017 (1436\%) e, um decréscimo de 15,59\% e 23,12 \% em 2016 e 2018. Analisando os casos de TB-MDR nota-se uma variação de 20 a 266, e média 165,3 casos. Observou-se também um aumento expressivo no número de TB-MDR entre 2010 e 2015 (1540\%) e, um decréscimo de 17,2\% e 32,3\% em 2016 e 2018 (Figura 1).

Figura 1: Número de casos tuberculose resistente e multirresistente no Brasil, 2009 a 2018.

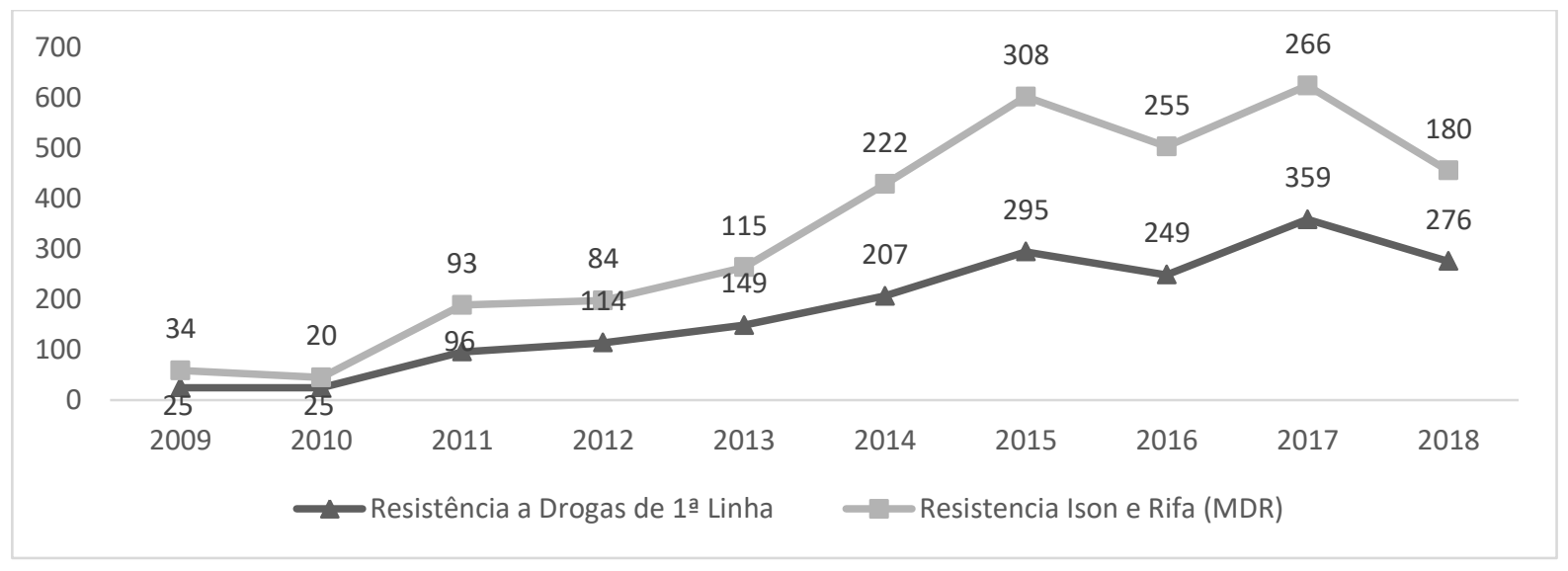

Fonte: Ministério da Saúde/SVS - Sistema de Informação de Agravos de Notificação - Sinan Net.

Em relação a distribuição dos casos de TB resistente de acordo com as regiões brasileiras, verifica-se um maior número de notificações de TB resistente a drogas de $1^{\text {a }}$ linha e TB-MDR na região Sudeste (n.1153/65,1\%) e Sul (n.233/13\%). No entanto, na região Sul o 
número de casos de TB-MDR foi superior ao de casos de resistência a drogas de $1^{\mathrm{a}}$ linha (Figura 2).

Figura 2: Número de casos de tuberculose resistentes entre as regiões brasileiras, 2009 a 2018.

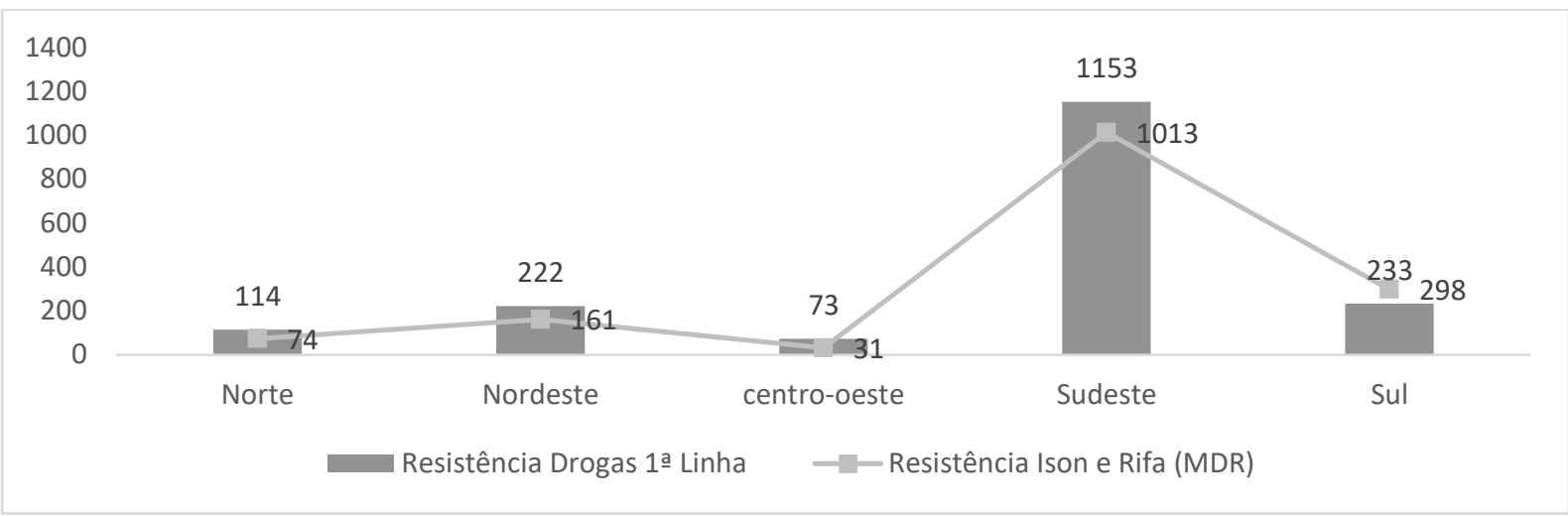

Fonte: Ministério da Saúde/SVS - Sistema de Informação de Agravos de Notificação - Sinan Net.

Analisando a prevalência média dos casos de TB-MDR, entre os últimos dez anos de acordo com as regiões brasileiras, verifica-se uma maior prevalência na região sudeste $(0,13$ casos $)$ e na região norte $(0,05)$ e menor na região Sul $(0,01)$ (Figura 3$)$, apesar dessa apresentar o segundo maior número de casos no geral.

Figura 3: Mapa epidemiológico da prevalência média da TB-MDR entre as regiões brasileiras, 2009 a 2018.

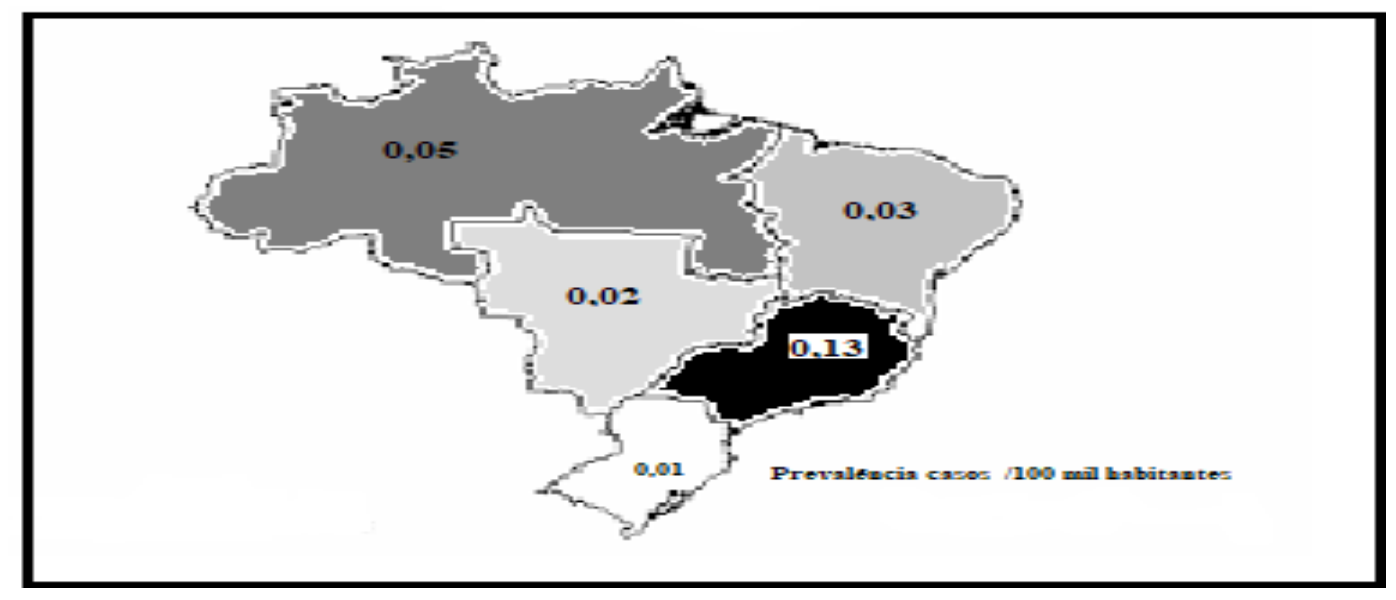

Fonte: Ministério da Saúde/SVS - Sistema de Informação de Agravos de Notificação - Sinan Net. 
Em relação aos dados sociodemográficos dos pacientes notificados, nota-se o predomínio da doença no sexo masculino (71,47 \%), na faixa etária entre 25-44 anos (41\%), com $1^{\circ}$ grau incompleto $(43,44 \%)$ e entre as raças branca $(37,6 \%)$ e parda $(36,84 \%)$, havendo diferenças estatisticamente significativas entre essas variáveis $(\mathrm{p}<0,001)$. Para a variável tipo de entrada $(\mathrm{p}<0,001)$, os casos novos $(45,97 \%)$ e de reingresso após abandono $(28,34 \%)$, apresentaram os maiores percentuais de caso (Tabela 1).

Tabela 1. Dados sociodemográficos dos pacientes com TB-MDR no Brasil, 2009 a 2018.

\begin{tabular}{|c|c|c|c|}
\hline Variáveis & Total & $\%$ & Valor de $p$ \\
\hline Total & 1577 & 100 & \\
\hline Sexo & & & $<0,001$ \\
\hline $\mathrm{F}$ & 450 & 28,53 & \\
\hline M & 1127 & 71,47 & \\
\hline Faixa Etária & & & $<0,001$ \\
\hline 0 a 14 & 18 & 1,14 & \\
\hline 15 a 24 & 256 & 16,23 & \\
\hline 25 a 34 & 391 & 24,79 & \\
\hline 35 a 44 & 365 & 23,15 & \\
\hline 45 a 54 & 274 & 17,37 & \\
\hline 55 a 64 & 200 & 12,69 & \\
\hline$\geq 65$ & 73 & 4,63 & \\
\hline Cor/Raça & & & $<0,001$ \\
\hline Ign/branco & 101 & 6,4 & \\
\hline Branca & 593 & 37,60 & \\
\hline Preta & 292 & 18,52 & \\
\hline Parda & 581 & 36,84 & \\
\hline Outros & 10 & 0,64 & \\
\hline Escolaridade & & & $<0,001$ \\
\hline Ign/Branco & 296 & 18,77 & \\
\hline Analfabeto & 60 & 3,80 & \\
\hline $1^{\circ}$ grau inc. & 685 & 43,44 & \\
\hline $1^{\circ}$ grau comp. & 66 & 4,19 & \\
\hline $2^{\circ}$ grau inc. & 275 & 17,44 & \\
\hline $2^{\circ}$ grau comp. & 104 & 6,59 & \\
\hline Ensino superior & 78 & 4,95 & \\
\hline Não se aplica & 13 & 0,82 & \\
\hline Tipo Entrada & & & $<0,001$ \\
\hline Caso Novo & 725 & 45,97 & \\
\hline Recidiva & 340 & 21,55 & \\
\hline Reing/abandono & 447 & 28,34 & \\
\hline Transferência & 65 & 4,14 & \\
\hline
\end{tabular}

Fonte: Ministério da Saúde/SVS - Sistema de Informação de Agravos de Notificação Sinan Net 
Analisando os dados clínicos dos pacientes notificados com TB-MDR no Brasil, nota-se que de forma geral, a maioria apresentava resistência adquirida (54\%), 24,85\% eram alcoolistas, 24,41\% tabagistas, 17,6\% eram portadores do vírus HIV, a maioria apresentava a forma pulmonar $(93,65 \%)$ e não realizavam o tratamento diretamente observado (TDO) (52,3\%). A proporção de casos que haviam evoluído à cura no momento da coleta dos dados, foi de somente $8,3 \%$ (Tabela 2 ).

Tabela 2. Dados epidemiológicos clínicos associados à resistência primária e adquirida, entre os pacientes com TB-MDR no Brasil, 2009 a 2018.

\begin{tabular}{|c|c|c|c|c|c|c|}
\hline \multirow[b]{2}{*}{ Variáveis } & \multirow{2}{*}{$\begin{array}{l}\text { Amostra } \\
\mathrm{n}(\%)\end{array}$} & \multicolumn{2}{|c|}{ Resistência primária } & \multicolumn{2}{|c|}{ Resistência adquirida } & \multirow[b]{2}{*}{$p^{*}$} \\
\hline & & $\mathrm{n}$ & $\%(95 \%$ IC) & $\mathrm{n}$ & $\%(95 \%$ IC) & \\
\hline Total & $1577(100)$ & 725 & $46,0(43,5-48,4)$ & 852 & $54,0(51,6-56,5)$ & \\
\hline Alcoolismo & & & & & & $<0,001$ \\
\hline Sim & $392(25,6)$ & 149 & $38,0(33,3-42,9)$ & 243 & $62,0(57,1-66,7)$ & \\
\hline Não & $1138(74,4)$ & 555 & $48,8(45,9-51,7)$ & 583 & $51,2(48,3-54,1)$ & \\
\hline Tabagismo & & & & & & 0,053 \\
\hline Sim & $385(25,3)$ & 162 & $42,1(37,3-47,1)$ & 223 & $57,9(52,9-62,8)$ & \\
\hline Não & $1138(74,7)$ & 502 & $44,1(41,3-47,0)$ & 551 & $48,4(45,5-51,3)$ & \\
\hline$H I V$ & & & & & & 0,002 \\
\hline Positivo & $247(17,6)$ & 90 & $36,4(30,7-42,6)$ & 157 & $63,6(57,4-69,3)$ & \\
\hline Negativo & $1156(82,4)$ & 547 & $47,3(44,5-50,2)$ & 609 & $52,7(49,8-55,6)$ & \\
\hline$T D O$ & & & & & & 0,121 \\
\hline Sim & $543(47,7)$ & 270 & $49,7(45,5-53,9)$ & 273 & $50,3(46,1-54,5)$ & \\
\hline Não & $596(52,3)$ & 269 & $45,1(41,2-49,2)$ & 327 & $54,9(50,9-58,8)$ & \\
\hline Forma & & & & & & 0,023 \\
\hline Pulmonar & $1477(96,1)$ & 664 & $45,0(42,4-47,5)$ & 813 & $55,0(52,5-57,6)$ & \\
\hline Extrapulmonar & $47(3,1)$ & 29 & $61,7(47,4-74,2)$ & 18 & $38,3(25,8-52,6)$ & \\
\hline Encerramento & & & & & & $<0,001$ \\
\hline Cura & $131(8,3)$ & 83 & $63,4(54,8-71,1)$ & 48 & $36,6(28,9-45,2)$ & \\
\hline Outros & $1446(91,7)$ & 642 & $44,4(41,9-47,0)$ & 804 & $55,6(53,0-58,1)$ & \\
\hline
\end{tabular}

Nota: * Teste Qui-Quadrado de Person.

$\mathrm{Na}$ associação dos aspectos epidemiológicos clínicos dos pacientes com TBMDR, em relação a resistência primária ou adquirida, foram encontradas diferenças 
estatisticamente significativas $(\mathrm{p}<0,005)$, para as seguintes variáveis: alcoolismo, HIV, forma da doença e situação de encerramento (Tabela 2).

Indivíduos alcoolistas (p 0,002; IC95\% 57,1-66,7), portadores do vírus HIV ( $\mathrm{p}<$ 0,001; IC95\% 57,4-69,3), com a forma pulmonar da doença (p 0,023; IC95\% 52,5-57,6), e outras formas de encerramento da doença ( $p<0,001$; IC95\% 53,0-58,1), apresentaram maiores percentuais de resistência adquirida. No entanto, os pacientes portadores da forma extrapulmonar (p 0,023; IC95\% 47,4-74,2) e que evoluíram à cura, apresentaram maiores percentuais de resistência primária ( $\mathrm{p}<0,001$; IC95\% 54,8-71,1) (Tabela 2).

\section{DISCUSSÃO}

Estudos demostram a fragilidade do sistema de saúde do Brasil em manejar eficientemente casos diagnosticados de TB, favorecendo o desenvolvimento de cepas resistentes ao tratamento. Tratamentos inadequados, dificuldades de acesso aos serviços de saúde e baixa adesão ao tratamento da TB estão entre as principais causas de TB-MDR. ${ }^{8}$

Esse aumento pode ser explicado também, pelo surgimento de outros bancos de dados de notificação, como o Sistema de Vigilância Epidemiológica da Tuberculose Multirresistente e o Sistema de Informação de Tratamentos Especiais da Tuberculose (SITETB), destinados ao acompanhamento e notificação de casos especiais de TB, como reações adversas ao tratamento, toxicidade, resistência ou comorbidades que inviabilizem o esquema básico. ${ }^{9}$

O número de casos de TB resistente a drogas de primeira linha, apresentou um decréscimo de 15,6\% em 2016 e 23,1\% em 2018. Enquanto os casos de TB-MDR diminuíram de 17,2\% em 2016 e 32,3\% em 2018. Este cenário justifica-se por um aumento ao acesso do Tratamento Diretamente Observado (TDO), meta do programa Brasil Livre da Tuberculose. ${ }^{10}$ O TDO é fundamental para o sucesso do tratamento por ser realizado de forma supervisionada. Esta estratégia viabiliza o aumento da adesão dos pacientes, estabelecimento de vínculo e maior descoberta das fontes de infecção, o que possibilita um aumento dos percentuais de cura e reduz o risco de transmissão da doença na comunidade. ${ }^{11}$

Em relação a distribuição dos casos de TB resistente de acordo com as regiões brasileiras, verifica-se um maior número de notificações de TB resistente a drogas de $1^{\text {a }}$ linha e TB-MDR na região Sudeste e Sul. No entanto, na região Sul o número de casos de TB- 
MDR foi superior ao de casos de resistência a drogas de $1^{\mathrm{a}}$ linha. Fato que pode estar relacionado ao nível de retratamento de pacientes nessas localidades, em decorrência do abandono ou recidiva da doença.

Analisando a prevalência média dos casos de TB-MDR, entre os últimos dez anos de acordo com as regiões brasileiras, nota-se uma maior prevalência na região sudeste $(0,13$ casos) e na região norte $(0,05)$.

Segundo o boletim epidemiológico da doença, as regiões com maior incidência e prevalência da doença são a região norte e nordeste do país. E as que apresentam as maiores taxas de abandono ao tratamento, região nordeste, sul e sudeste. ${ }^{10}$ Fatores que podem contribuiu para maior prevalência de casos de TB-MDR nessas regiões.

Apesar dos tratamentos serem disponibilizados gratuitamente na rede pública, a dispensa das medicações e o seguimento do tratamento são realizados em locais distintos. A longa duração e os efeitos colaterais do tratamento também favorecem a falta de adesão ${ }^{12}$ e consequentemente o aumento das cepas resistentes.

Em relação aos dados sociodemográficos dos pacientes analisados, nota-se o predomínio da doença no sexo masculino (71,47 \%), na faixa etária entre 25-44 anos (41\%), com $1^{\circ}$ grau incompleto $(43,44 \%)$, entre as raças branca $(37,6 \%)$ e parda $(36,84 \%)$, havendo diferenças estatisticamente significativas entre essas variáveis $(\mathrm{p}<0,001)$, corroborando com os dados encontrados na literatura. O predomínio da doença entre adultos jovens, na faixa etária produtiva e, com baixa escolaridade, confirmam a importância socioeconômica dessa infecção sobre os indivíduos, reforçando a relação da enfermidade com a pobreza e o baixo acesso a saúde e educação. ${ }^{10}$

A pobreza, muitas vezes, está relacionada ao adoecimento por $\mathrm{TB}$, porquanto indivíduos com baixa renda têm cada vez menos acesso à informação. Além disso, os grupos populacionais agrupados por idade, escolaridade, raça e sexo que estão condicionados a essa situação de vida e saúde são suscetíveis ainda mais ao adoecimento por tal moléstia. ${ }^{13}$

Para a variável tipo de entrada $(\mathrm{p}<0,001)$, os casos novos $(45,97 \%)$ e de reingresso após abandono (28,34\%), apresentaram os maiores percentuais de caso entre os pacientes com TB-MDR. Estudo realizado no Espírito Santo por Fregona et al. ${ }^{14}$ também verificou maior percentual de casos novos entre os pacientes com a forma resistente. 
Analisando os dados clínicos dos pacientes com TB-MDR no Brasil, 54\% apresentava resistência adquirida e $46 \%$ resistência primária. No entanto, no estudo realizado por Savioli, Morrone e Santoro, ${ }^{15}$ foi detectada taxa de resistência primárias de $36 \%$ e adquirida de $64 \%$. Dantas et al., ${ }^{16}$ relataram casos de TB-MDR por transmissão primária em 20 a 30\% dos doentes. É de suma importância a diferenciação entre resistência primária e resistência adquirida, pois pode haver implicações na estratégia programática no controle da transmissão do bacilo multidrogarresistente. ${ }^{15}$

O presente estudo também demonstrou que Indivíduos alcoolistas ( $p$ 0,002; IC95\% 57,1-66,7), portadores do vírus HIV ( $p<0,001$; IC95\% 57,4-69,3), com a forma pulmonar da doença (p 0,023; IC95\% 52,5-57,6), e outras formas de encerramento da doença, como por exemplo o abandono ( $\mathrm{p}<0,001$; IC95\% 53,0-58,1), apresentaram maiores percentuais de resistência adquirida. No entanto, os pacientes portadores da forma extrapulmonar (p 0,023; IC95\% 47,4-74,2) e que evoluíram à cura, apresentaram maiores percentuais de resistência primária $(\mathrm{p}<0,001$; IC95\% 54,8-71,1)

Estudos constataram que o consumo de álcool altera significativamente a resposta imune, aumentado a susceptibilidade a doenças respiratórias, assim como a infecção pelo HIV. ${ }^{17}$ Também, foi demonstrado que o tabagismo está associado há um insucesso de tratamento, pois o tempo de conversão da cultura de escarro de positiva para negativa entre os fumantes é superior que os não fumantes após o segundo mês de tratamento. ${ }^{18}$

De acordo com Souza, Silva e Miranda, ${ }^{19}$ o abandono do tratamento da tuberculose ainda é um desafio que implicará significativamente o controle da patologia, mediado por fatores passíveis de intervenção, entre eles: a falta de informação do paciente sobre a doença; a falta de informação da família; o uso de álcool e de drogas ilícitas; as barreiras sociais, econômicas, demográficas e culturais; a escolaridade; os problemas inerentes ao medicamento e o tratamento diretamente observado (TDO). A resistência aos medicamentos usuais é outro condicionante para eliminar a tuberculose. A esse respeito, Fontes et al. ${ }^{13}$ propõem a inclusão de tratamentos com regimes mais simplificados, mais curtos e menos tóxicos, que poderão diminuir os casos de resistência.

Desta forma, o conhecimento do espaço é de relevante importância na análise das relações de saúde com o ambiente, fornecendo subsídios à gestão e aos serviços para as ações 
de controle, podendo provocar mudanças significativas no quadro de morbimortalidade de uma sociedade. ${ }^{20}$

A principal limitação desse tipo de estudo, foi o uso de dados secundários, pois esses podem interferir na análise, sobretudo pela baixa completude dos registros. Nesse sentido, é indispensável garantir a sensibilidade e a confiabilidade dos dados do SINAN para a sua utilização com segurança por pesquisadores, gestores e profissionais da saúde de igual forma no território nacional. ${ }^{12}$

Diante do proposito de uma efetiva redução dos casos novos de TB-MDR, tornase necessário o desenvolvimento de um processo que identifique áreas com diferentes necessidades e ações. Desta forma, o programa de controle da tuberculose irá investir na busca de pacientes sintomáticos respiratórios e na identificação de população de risco para a tuberculose latente. A identificação e o fortalecimento do uso racional dos medicamentos (esquemas padronizados e adesão ao tratamento), acarretará uma real redução do número de casos novos. $^{21}$

\section{CONCLUSÃO}

O número de casos de tuberculose resistente e multirresistente aumentou expressivamente entre 2010 e 2017. Teve predomínio no sexo masculino, na faixa etária entre 25-44 anos, com $1^{\text {o }}$ grau incompleto, nas raças branca e parda. Os casos novos e de reingresso após abandono, apresentaram os maiores percentuais. Indivíduos alcoolistas, portadores do vírus HIV, com a forma pulmonar, e com abandono do tratamento, tiveram maiores percentuais de resistência adquirida. No entanto, portadores da forma extrapulmonar e que evoluíram à cura, apresentaram maiores percentuais de resistência primária. Conclusão: A tuberculose se mantém, como uma doença de alta incidência/prevalência em nosso país e, que apesar dos investimentos o percentual de casos de resistência permanece altos, trazendo importantes repercussões clínicas e epidemiológicas.

\section{REFERÊNCIAS}

1- BRASIL. Ministério da Saúde. Secretaria de Vigilância em Saúde. Departamento de Vigilância das Doenças Transmissíveis. Manual de Recomendações para o Controle da Tuberculose no Brasil. 2. ed. Brasília: Ministério da Saúde, 2019. Disponível em: 
http://bvsms.saude.gov.br/bvs/publicacoes/manual_recomendacoes_controle tubercul ose_brasil_2_ed.pdf. Acesso em:_05 de maio, 2020.

2- MENDES, Marcos Ramon Ribeiro dos Santos et al. Situação sóciodemográfica da tuberculose multirresistente no estado do Piauí, 2001-2012. Revista Interdisciplinar, Teresina, v. 7, n. 1, p. 8-16, jan-mar, 2014.

3- HOAGLAND, Daniel $\mathrm{T}$ et al. New agents for the treatment of drug-resistant Mycobacterium tuberculosis. Advanced Drug Delivery Reviews, Amsterdam, v. 1, n. 102, p. 55-72, jul, 2016.

4- GYGLI, Sebastian M et al. Antimicrobial resistance in Mycobacterium tuberculosis: mechanistic and evolutionary perspectives. FEMS Microbiology Reviews, Cambridge, v. 41, n. 3, p. 354-373, maio, 2017.

5- SONG, Wan-mei et al. Primary drug resistance of mycobacterium tuberculosis in Shandong, China, 2004-2018. Respiratory Research, New York, v. 20, n. 1, p. 223, out, 2019.

6- ARBEX, Marcos Abdo et al. The challenge of managing extensively drug-resistant tuberculosis at a referral hospital in the state of São Paulo, Brazil: a report of three cases. Jornal Brasileiro de Pneumologia, São Paulo, v. 41, n. 6, nov-dez, 2015.

7- WHO. World Health Organization. Global Tuberculosis Report - 2019. Genebra: World Health Organization; 2019. Disponível em: https://apps.who.int/iris/bitstream/handle/10665/329368/9789241565714eng.pdf?ua=1. Acesso em 20 fevereiro, 2020.

8- ROCHA, Paula Souza da Silva.; DA SILVA, Marcos Valério Santos.; DE ANDRADE, Marcieni Ataíde. Série histórica da tuberculose resistente a múltiplos medicamentos (TB-MR) no estado do Pará, Brasil, 2005-2014. Revista de Epidemiologia e Controle de Infecção, Santa Cruz do Sul, v. 7, n. 2, p. 96-100, abr, 2017.

9- DE ALMEIDA, Manoel Guedes.; BARBOSA, Débora Regina Marques.; ALMEIDA, Dhony Ferraz da Silva. Epidemiologia e distribuição espacial da tuberculose multirresistente (TBMR) no Brasil notificada através do SINAN, 2008-2012. Revista de Epidemiologia e Controle de infecção, Santa Cruz do Sul, n. 3, v. 4, p. 117-122, out-dez, 2013. 
10- BRASIL. Ministério da Saúde. Implantação do Plano Nacional pelo Fim da Tuberculose como Problema de Saúde Pública no Brasil: primeiros passos rumo ao alcance das metas. Boletim epidemiológico, Brasília, v. 49, n. 11, mar, 2018.

11-COSTA, Hosana Mirelle Goes e Silva et al. A importância do trabalho em equipe na efetivação do tratamento diretamente observado em tuberculose. Revista de Enfermagem UFPE on line, Recife, v. 10, n. 4, p. 1202-1209, abr, 2016.

12-GASPAR, Renato Simões et al. Análise temporal dos casos notificados de tuberculose e de coinfecção tuberculose-HIV na população brasileira no período entre 2002 e 2012. Jornal Brasileiro de Pneumologia, São Paulo, v.42, n.6, nov-dez, 2016.

13-FONTES, Giuliano José Fialho et al. Perfil epidemiológico da tuberculose no Brasil no período de 2012 a 2016. Revista Brasileira de Educação e Saúde, Pombal, v. 9, n. 1, p. 19-26, jan-mar, 2019.

14- FREGONA, Geisa et al. Fatores associados à tuberculose resistente no Espírito Santo, Brasil. Revista de Saúde Pública, São Paulo, v. 51, p. 1-11, 2017.

15-SAVIOLI, Márcia Telma Guimarães.; MORRONE, Nelson.; SANTORO, Ilka. Resistência bacilar primária em tuberculose multidrogarresistente e fatores preditivos associados à cura, em um centro de referência da cidade de São Paulo. Jornal Brasileiro de Pneumologia, São Paulo, v. 45, n. 2, e20180075, mar, 2019.

16- Dantas NG, Suffys PN, Carvalho W da S, Gomes HM, de Almeida IN, de Assis LJ, et al. Genetic diversity and molecular epidemiology of multidrug-resistant Mycobacterium tuberculosis in Minas Gerais State, Brazil. BMC Infectious Diseases, New York, v. 15, n. 306, p. 1-11, ago, 2015.

17-MOLINA, Patricia E et al. Focus on: Alcohol and the immune system. Alcohol Research \& Health, Michigan, v. 33, n.1-2, p. 97-108, 2010

18-MACIEL, E L et al. Smoking and 2-month culture conversion during antituberculosis treatment. International Journal of Tuberculosis of Lung Disease, Paris, v. 17, n. 2, p. 225-8, fev, 2013.

19-SOUZA, Adélia Camily Silva de.; SILVA, Maria Lucia Santos Jacinto.; MIRANDA, Lays Nogueira. Dificuldades na adesão do plano de tratamento pelo paciente com tuberculose. Cadernos de Graduação Ciências Biológicas e de Saúde, Maceió, v. 4, n. 2, p.297-311, fev, 2017. 
20- YAMAMURA, Mellina et al. Epidemiological characteristics of cases of death from tuberculosis and vulnerable territories. Revista Latino Americana de Enfermagem, Ribeirão Preto, v. 23, n. 5, p. 910-8, set-out, 2015.

21- Augusto, Cláudio José et al. Características da tuberculose no estado de Minas Gerais entre 2002 e 2009. Jornal Brasileiro de Pneumologia, São Paulo, v.39, n.3, p.357-364, maio-jun, 2013. 